Article

\title{
1, 2, 3, Many-Perceptual Integration of Motif Repetitions
}

\author{
Mikhail Katkov *(D, Hila Harris and Dov Sagi \\ Department of Neurobiology, Weizmann Institute of Science, Rehovot 76100, Israel; \\ Hila.Harris@gmail.com (H.H.); dov.sagi@weizmann.ac.il (D.S.) \\ * Correspondence: mikhail.katkov@gmail.com; Tel.: +972-8934-4324
}

Received: 18 October 2018; Accepted: 20 November 2018; Published: 21 November 2018

check for updates

\begin{abstract}
It is generally assumed that the initial integration of visual information is limited in its spatial extent. Of particular interest is the extent to which image symmetries are detected and integrated. Here we studied the spatial extent of visual integration in textures constructed from wallpaper symmetry groups. Using tools from statistical physics, we obtained images ranging from symmetric ones to completely random ones, whereas the textural elements were of the same quality. Results show that the psychometric curves for $3 \times 3$ motif repetitions are similar to those of images having more repetitions, whereas an equivalent physical scaling of the images does not alter the performance.
\end{abstract}

Keywords: order; visual texture; symmetry; phase transition

\section{Introduction}

Early stages of visual information processing in the human brain are well characterized in terms of local processes having oriented receptive fields [1,2]. It is believed that the later processing stages integrate local information into global percepts. However, the brain mechanisms underlying this integration are much less well understood. One well-studied class of visual tasks where spatial integration is of importance is the texture-discrimination task. In the domain of texture vision, it has been suggested that the ability to discriminate between textures is determined by a set of fixed measurements performed on the presented image (statistics) [3,4]. Conceptually, two types of measurement were suggested: (1) The average value of some local image transformation [5,6] and (2) global computation [7,8]. Examples of the former are mean intensity, variance, and kurtosis, where local transformations are pointwise power functions of the intensity, which are averaged. An example of the latter is an image autocorrelation function, where distant parts of an image are involved in computations. We were interested in determining the extent of the local integration in texture discrimination.

We considered a subclass of visual textures consisting of perfectly repeating patterns [9-11]. It is known that two-dimensional repeating patterns form wallpaper groups [12]: 17 two-dimensional symmetry groups (two-dimensional crystallographic groups). Each symmetry group represents a specific combination of symmetries present in each pattern that belongs to this symmetry group. For example, symmetry group P1 consists of translations only, meaning if an image belongs to this symmetry group, one can find two non-collinear translation vectors that will map an image to itself; furthermore, any other transformation (except multiple applications of translations) results in a different image (at least some part of the image pair differs in pixel intensity). Symmetry group P2 consists of translations and 2-fold rotations, that is, the image belongs to P2, if in addition to translation, one can find an axis where a rotation by $180^{\circ}$ maps the image to itself. Other groups have 
different combinations of symmetries. In total, 17 combinations of symmetries are consistent with two-dimensional translations.

Here, we set out to measure the human ability to detect and to discriminate between deviations in symmetry. To this end, images were created with different degrees of symmetry in a way that can be characterized by a single parameter. Mathematics, although dealing with perfect symmetry, does not offer a measure of graded symmetry. One method used in the past to generate such a continuum between perfect symmetry and its complete absence was adding white noise to pixel values [13]. However, this procedure degrades the quality of the initial image transformations within the primary visual cortex in a way that may affect the integration mechanisms. We have recently suggested [14] a method for perturbing perfectly repeating patterns without reducing the quality of low-level representation in any significant way.

More specifically, in this work we used theoretical tools developed in statistical physics to control and quantify the degree of order (graded symmetry) in synthesized images. Next, the intuition underlying these tools will be briefly presented. Many physical systems undergo a phase transition, a transition between ordered and disordered states when the temperature changes. For example, at low temperatures, atoms in crystals are almost perfectly arranged on a lattice, whereas at high temperatures crystals melt, forming a liquid state where the atoms are randomly positioned, with an abrupt transition between ordered and disordered states taking place at a specific temperature (first-order phase transition). Alternatively, the order may gradually increase with a decreasing temperature (second-order phase transitions), like in ferrimagnets, where it disappears completely at a specific temperature (phase transition temperature, critical temperature). It is important here that (1) for the second-order phase transitions, no trace of symmetry related to the ordered phase is present above the critical temperature, and that a graded degree of symmetry is present close to phase transitions [15]; (2) the degree of symmetry can be quantified by an order parameter [16]; and (3) one can simulate artificial physical systems at arbitrary thermodynamic temperatures using statistical physics tools and one can visualize the equilibrium state.

Technically, simple models of ferrimagnets are usually described as a collection of interacting spins placed on a lattice (considered as small magnets) that tend to co-align [17]. At low temperatures, most spins point in one direction, and eventually, given sufficient time, all spins take one of the two directions. With increasing temperature, more spins flip locally, eventually having their direction randomized at high temperatures, leading to zero net magnetization. The order parameter for this system is net magnetization, i.e., if one assigns +1 for spin up and -1 for spin down, the order parameter is the absolute value of the mean of all values in the lattice. For very large systems at high temperatures, the order parameter is a small number approaching zero, when the system size is increased, whereas below the transition point, the order parameter is gradually increased, approaching a value of one at very low temperatures. One can visualize this system using dots, Gaussian blobs, or Gabor patches placed on the vertices of a lattice instead of spins and by replacing spin direction (up/down) using image properties such as light intensity, contrast (high/low), or by color. The order parameter is a mean value of, possibly, a more complicated property computed locally (e.g., orientation), reflecting the global symmetry in the ordered phase in such a way that when the system is consistent with global symmetry the order parameter approaches a value of one and when the system is in random configurations it vanishes. Importantly, the order parameter can usually be considered as a first-order statistic of some local measurements, which in general is consistent with some accounts in texture perception [5,6]. The locally computed values form an "order parameter field".

Note that phase transition is associated with changes in symmetry in the system [15], and therefore, the sensitivity to symmetry in the human visual system should be manifested around phase transitions, even if the activation of low-level features is perfect. Recently, we have tested the hypothesis that the human visual system is sensitive to order-disorder transitions [14]. More specifically, we created two-dimensional artificial physical systems similar to the Ising model described above, mapped into visual stimuli. Using different patterns of interactions between neighboring elements, we obtained 
a variety of ordered states with different organizations. We then simulated those artificial physical systems at different thermodynamic temperatures to obtain equilibrium configurations, which were visualized by assigning different luminance levels to different local states. We found that the order-observer, defined as an observer that measures first-order statistics for a measure corresponding to specific symmetries, provided a better fit to the data than did the ideal observer, having a complete knowledge of the texture model used in the task. Here we studied how many repetitions are effectively integrated by the brain mechanisms for order detection and investigated whether computation depends on the physical size of an image. We used artificial physical systems with symmetries of the wallpaper groups-2-dimensional crystallographic groups $[12,18]$. The size of the presented stimuli and the number of motif repetitions were varied and it was found that the integration window of human observers is equivalent to $3 \times 3$ repetitions of the motif. Moreover, the performance was invariant to the physical scaling of the image. This finding indicates that if cross-correlation or first-order statistics-based mechanisms are involved in the perception of repetition, they are only applied to a limited extent.

\section{Materials and Methods}

Target images consisted of Gaussian blobs, where each was assigned one of three different amplitudes then placed on the vertices of a lattice. Therefore, the image is uniquely defined by 3 integer values $(0,1$, and 2$)$ associated with each vertex, $k$, on a lattice. Consequently, the image is completely described by a two-dimensional array $\operatorname{Im}(k) \in[0,1,2]$. Therefore, the Gaussian blobs can be replaced by any other 3-valued image triplets. In order to manipulate the arrangement of Gaussian blobs within the image, we sampled image configurations from Boltzmann distributions of different temperatures using a Gibbs sampler.

Technically, target stimuli were generated using the following Boltzmann distribution:

$$
P(\operatorname{Im})=\frac{1}{Z} e^{-\frac{U(\operatorname{Im})}{T}} .
$$

Here, $P(\operatorname{Im})$ is the probability of a particular image Im occurrence, $\mathrm{Z}$ is a normalization constant, $U(\operatorname{Im})$ is the potential defining the ordered configurations, and $T$ is the thermodynamic temperature, controlling the amount of thermal noise introduced into the system. When $T$ increases, the argument of the exponent decreases, practically vanishing at large $T$ values, leading to a uniform distribution over all images. At low $T$ values, small differences in $U(\operatorname{Im})$ are amplified, such that only images leading to minimal values of $U(\mathrm{Im})$ can be realized. The target stimuli were constructed based on the wallpaper symmetry groups (see Section 2.1); therefore, the potential $U$ was consistent with the chosen symmetry group (Section 2.1). Consequently, at low temperatures, only images with symmetry of the potential U were generated. We employed Gibbs sampling (a standard technique to generate thermal equilibrium configurations) to generate system configurations for finite temperatures (see Section 2.3).

Conceptually, our stimuli can be understood as a collection of textural elements with identities 0 , 1 , and 2 placed at the vertices of a lattice. As such, any set of textural elements or images can be used instead of the Gaussian blobs used here, the only requirement is a unique association with an identifier. Equation (1) defines the probability of a specific arrangement of these textural elements within a texture (lattice). At low temperatures, at thermal equilibrium, this arrangement will be consistent with the potential (U) symmetry, since all non-symmetric configurations would have exponentially small probabilities. Conversely, at high temperatures the probability of obtaining any configuration is practically constant, since the argument in the exponent vanishes for all configurations. The standard algorithm used to simulate thermal equilibrium for an arbitrary complicated system, where local conditional probabilities are unknown, is the Gibbs sampling algorithm. 


\subsection{Wallpaper Groups}

The wallpaper groups are a mathematical classification of two-dimensional repetitive patterns. The wallpaper groups consist of 17 distinct symmetry groups, each containing two linearly independent translations and other symmetries consistent with those translations. Two translation vectors define a "cell" with a finite area that tiles the whole 2D space. The cell may have an internal structure defined by the combination of 6 symmetries: 4 Rotations $\left(180^{\circ}, 120^{\circ}, 90^{\circ}\right.$, and $\left.60^{\circ}\right)$, a reflection, and a glide reflection. For example, in the P1 group the cells have no internal structure and an arbitrary non-symmetric image is repeated by translation; in the P2 group, the cells only have second order centers of rotation (the image is identical to itself rotated by $360^{\circ} / 2=180^{\circ}$ around these centers). A Bravais lattice is defined by a set of points in the vertices of cells tiling the 2D space [19]. There are five different Bravais lattices: Hexagonal, square, rectangular, rhombic, and oblique.

\subsection{Target Image Potentials}

The potential $U(\mathrm{Im})$ was defined on the Bravais lattice corresponding to the particular symmetry group. For each vertex $k$ of the lattice, a state value $\operatorname{Im}(k)$ was defined, similar to the spin values in the Ising model, but with 3 different values: 0,1 , and 2 (i.e., $\operatorname{Im}(k) \in[0,1,2]$ ). The translation vectors (defining the cell) were chosen to be 7-9 unit vectors of the lattice and the size of the lattice was around $8 \times 8$ cells. The numbers were slightly different for different groups. The global potential in the Boltzmann distribution was

$$
U(\operatorname{Im})=\sum_{k} U_{k}(\operatorname{Im}),
$$

where $U_{k}(\operatorname{Im})$ is the local potential at vertex $k$, and the summation is performed over all vertices in the lattice. The local potential, in turn, is

$$
U_{k}(\operatorname{Im})=\sum_{s \in S \prime} U_{s}(k, \operatorname{Im})
$$

where $U_{s}(k, \operatorname{Im})$ is a local potential consistent with the specific symmetry, $S$, such as rotation, translation, reflection, and glide reflection. To reduce the computational burden, the subset $S /$ of all possible symmetries in the image was used, which led to the generation of images with correct symmetries at low temperatures. For example, only nearby cells were used for implementing translation symmetries; mostly the cell itself and sometimes neighboring cells were used to define the potential for the rotation, reflection, and glide reflection symmetries.

Since the images used here are defined on a finite lattice, whereas the translation symmetry is defined on the whole two-dimensional plane, one needs to deal with the vertices on the boundary of the image. Thus, cyclic boundary conditions were employed. In other words, repetition of the whole image in the 2D plane was assumed, and therefore, vertices on the right border and left (similarly, the top and bottom) border are effectively stitched in a single infinite, strictly repeating image.

The symmetry potential itself was defined for pairs of vertices $-(k, p)$, where $p$ is a vertex index to where $k$ is mapped by the symmetry transformation. Formally, if we let $T_{s}$ be a symmetry transformation used for mapping vertices' coordinates to vertices' coordinates according to symmetry $s$, then

$$
U_{s}(k, \operatorname{Im})=\beta_{s}\left(1-\delta_{\operatorname{Im}(k), \operatorname{Im}\left(T_{s}(k)\right)}\right),
$$

where $\beta_{s}$ is a symmetry-specific constant that was fixed throughout the experiment, $\delta$ is a Kronecker delta, and $\operatorname{Im}(k)$ is a state value at location $k$ on the lattice. In other words, the potential is zero when the state values are the same at locations related by the symmetry, where otherwise they are the constant $\beta_{s}$. Examples of $T_{S}$ include two translations in the direction of the lattice basis by the size of the cell, a reflection about a specific axis, a glide reflection about a specific axis with a translation vector along that axis, rotation around a specific point by a specific angle; the possible angles are $60^{\circ}$, $90^{\circ}, 120^{\circ}$, and $180^{\circ}$ clockwise and counterclockwise. The value of $\beta_{s}$ defines the relative strength 
of one symmetry with respect to other symmetries in Equation (3) at intermediate temperatures. In the experiments, $\beta_{s}$ was set (by eye) in such a way that it allowed us to have different symmetries generated by a specific group expressed within a useful range of temperatures, thus improving the efficiency of the data collection. The specifics and the choice of $\beta_{s}$ values for each symmetry can be found in the generation program [20].

\subsection{Gibbs Sampler}

To make an inference for complex systems such as Equation (1), a Gibbs sampling technique is usually used [21]. Basically, Gibbs sampling consists of choosing one location, computing the probability of state values given the rest of the image, and finally drawing the state value from the computed distribution, repeating this computation for all locations for many iterations. It is known that in the limit of infinite iterations the resulting image is drawn from the desired distribution, except for some exotic potentials. To save computation time, we split the lattice onto groups of vertices and updated all locations in one group simultaneously. The vertices in the one group were never used to compute state value distributions for vertices in the same group, i.e., the state value distributions for vertices from one group were dependent only on the vertices from other groups. All groups were updated sequentially. This procedure was repeated 1000 times and the final state arrangements were stored in the database. This technique is usually referred to as colored Gibbs sampling [22].

\subsection{Stimulus Arrangement}

The stimulus display consisted of four images placed in four quadrants of a visual field. The rest of the screen was gray with a luminance level of $15 \mathrm{~cd} / \mathrm{m}^{2}$. Each image consisted of Gaussian blobs placed on the vertices of a Bravais lattice with one of three amplitudes: 27,36 , and $50 \mathrm{~cd} / \mathrm{m}^{2}$, corresponding to the state value $\operatorname{Im}(k)$ at the given location $k$. Therefore, the low-level representations (Gaussian blobs) were identical for all images. The generated target was presented in a randomly chosen quadrant, whereas in the other three quadrants the state values were drawn, independently, from a uniform distribution (corresponding to an infinite temperature).

In Experiment 1 a full-size generated lattice was presented, whereas in Experiment 2 the presented images were either full size or cropped to the size of $1 / 2,1,2$, and 3 cells. In some conditions the cropped images were physically scaled on the screen by a factor of 2 .

Stimuli were viewed at a distance of $1 \mathrm{~m}$.

\subsection{Procedures}

Human observers initiated each trial by pressing the "spacebar" on a computer keyboard. After $300 \mathrm{~ms}$ of gray screen $\left(15 \mathrm{~cd} / \mathrm{m}^{2}\right)$, a stimulus was flashed for 10 consecutive video frames $(120 \mathrm{~Hz}$ refresh rate, $\approx 83 \mathrm{~ms})$, followed by a gray screen $\left(15 \mathrm{~cd} / \mathrm{m}^{2}\right)$ that remained until a response was provided. Observers were requested to report which quadrant of the visual display contained an image that differed in its appearance from the other three images (see Section 2.4 above). This type of response method is referred to as a four-alternative forced choice (4AFC) method. It is a forced-choice paradigm in the sense that observers have to provide a response even when they perceive all quadrants as equal. The response was reported by pressing one of the four keys " 4 ", " 5 ", " 1 ", and " 2 " on the numeric keypad of a computer keyboard. The arrangement of the response keys was directly mapped to the reported quadrants. Auditory feedback was provided to indicate a wrong response at the end of the trial.

In Experiment 1, a staircase method was employed to explore a large range of parameters in order to identify the active range of a psychometric function. In the staircase method the stimulus presented on a given trial depends on the history of the observer's performance. This method is designed for a quick estimation of the threshold. Here, trials from several "2-up/1-down" and "3-up/1-down" staircases were randomly intermixed. After a wrong response, the target temperature was decreased (this was done to increase order which is easier to discriminate than the inverse). 
The target temperature was increased after two or three consecutive correct responses, depending on the staircase type (2-up/1-down or 3-up/1-down staircase, respectively). With this procedure, the presented targets statistically tend to be concentrated around the perceptual threshold. Trials with images from one symmetry group were blocked together. In Experiment 2, 10 different temperatures were chosen, based on Experiment 1, spanning the active range of psychometric functions. Trials with images from different symmetry groups were randomly mixed.

Three sessions of $20 \mathrm{~min}$ each were performed in a single day, with a 15-min break between sessions. Observers performed several days of experiments.

\subsection{Human Observers}

This study was approved by the Weizmann Institute of Science Ethics Committee and the Helsinki Committee. Ten human observers with normal or corrected-to-normal vision participated in the experiment. Before experimentation all observers provided their informed consent, under the approved Declaration of Helsinki.

Nine observers (ages 20-42 years) with normal or corrected-to-normal vision participated in both experiments. Eight college students were recruited using advertisements. One observer participated in both experiments.

\subsection{Data Format}

The program for stimulus generation and visualization is available at https://github.com/ mkatkov/WallpaperColoredGibbsSampler (DOI:10.5281/zenodo.1420131). The stimuli used are available at https://osf.io/84mvb/ (DOI:10.17605/OSF.IO/84MVB). The data from Experiments 1 and 2 are presented in the supplementary material as MATLAB@ data files. All figures in the current contribution can be reproduced using a data analysis MATLAB@ script, given that the presentation program and image databases are downloaded and installed according to the instructions in the software package.

Following is the data format for Experiment 1. Each MATLAB@ data file corresponds to experiments performed by one human observer. The fields in the file are as follows:

- "betaIdx", randomized beta indexes; each index refers to the image index in the image database for the corresponding symmetry group.

- "groupList", a list of classes corresponding to the presented symmetry group.

- "groupPermutation", a random permutation of groups used in the presentation program.

- "trialData", a structure with records related to each performed trial. It consists of a cell array with an entry for each group. Each cell array entry is a structure with the following fields:

- "trialOnset", start of trials (Linux timestamp).

- "reportTime", time of report (Linux timestamp).

- "report", quadrant observer reported to have a different texture.

- "presentationQuadrant", quadrant where the target image was presented.

- "beatIdx", beta index in the image database (related to the thermodynamic temperature).

- "imgIdx", index of the image in the database with the same thermodynamic temperature.

Following is the data format for Experiment 2. Each MATLAB@ data file corresponds to experiments performed by one human observer. The fields in the file are as follows:

- "beatIdx", 10 thermodynamic temperatures were used in this experiment; this variable shows what thermodynamic temperature index was planned in this trial.

- "curStim", stimulus to be presented. The number shows the total number of trials performed by a human observer.

- "groupList", list of groups used in the experiment. 
- "groupIdx", index of groups in the "groupList" of the image planned for the current trial

- "sizeIdx", enumerated conditions for the planned current trial:

- $1 \times 1$ cells.

- $2 \times 2$ cells.

- $3 \times 3$ cells.

- full size image.

- full size image.

- $1 / 2 \times 1 / 2$ cells magnified twice.

- $1 \times 1$ cells magnified twice.

- $\quad 2 \times 2$ cells magnified twice.

- "trialData", a structure with records related to each performed trial. It consists of a cell array with an entry for each group. Each cell array entry is a structure with the following fields:

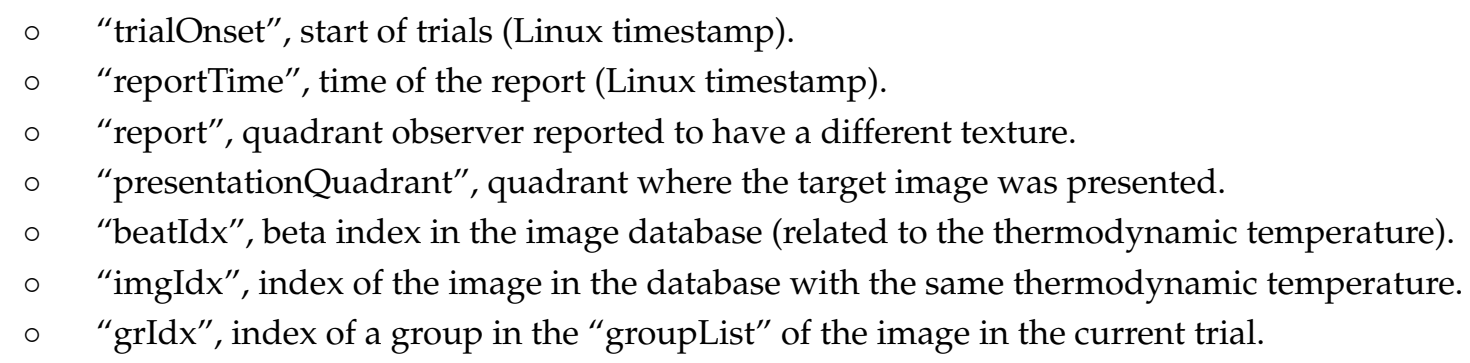

\section{Results}

Two experiments were performed. In Experiment 1 we obtained psychometric functions describing the correct discrimination (25\%-100\%). In Experiment 2 we used the results from Experiment 1 to define a set of 10 thermodynamic temperatures with performances spanning the active part of the psychometric function and we tested several conditions using a constant stimulus design to obtain better precision.

\subsection{Experiment 1}

In Experiment 1, all 17 wallpaper symmetry groups were presented to the observers. Since the potentials used to generate images have an arbitrary magnitude, the informative range of the psychometric function needs to be identified first. A mixture of adaptive processes was employed to estimate the effective range. The results of this experiment are presented in Figure 1. Each panel shows the performance of six observers as a function of an inverse temperature for a single symmetry group. These results were obtained by averaging the performances on 20 consecutive temperatures; therefore, there is an intrinsic variability due to the small sampling size. However, the psychometric curves for different observers are quite consistent, where temperature thresholds are approximately equal, considering the width of the psychometric functions. This observation suggests that the different observers share a similar mechanism of order perception. 

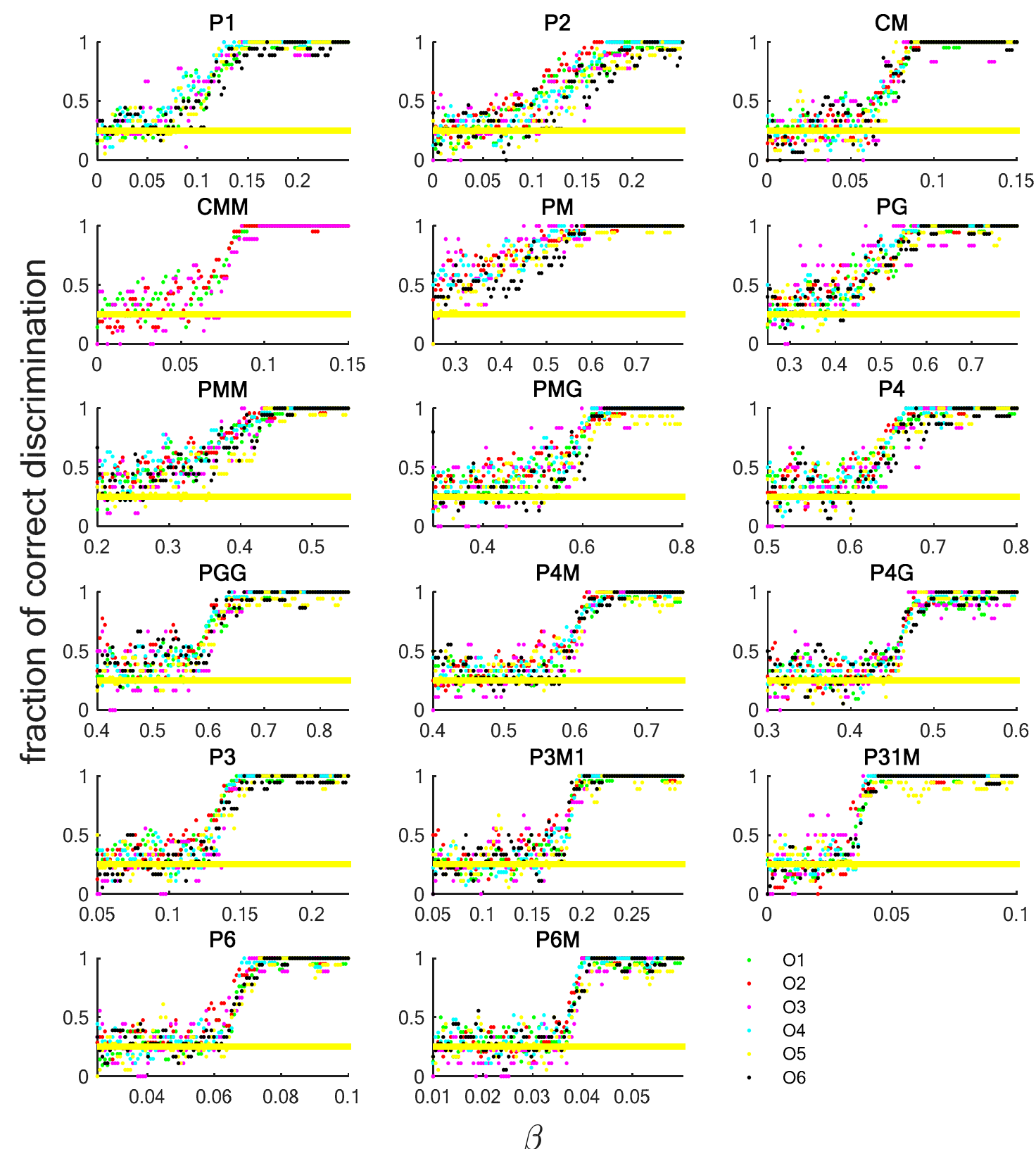

$\cdot \quad \mathrm{O} 1$
$\cdot \quad \mathrm{O} 2$
$\cdot \quad \mathrm{O} 3$
$\cdot \quad \mathrm{O} 4$
$\cdot \quad \mathrm{O5}$
.$\quad \mathrm{O} 6$

Figure 1. Results of Experiment 1 for 6 human observers O1-O6. The 17 panels correspond to the 17 symmetry groups, each showing the performance of six human observers (shown as different colors) as a function of inverse temperature $\beta$. The group name is shown in the panel title. The performance was measured as the mean value of correct responses for 20 consecutive temperature levels obtained using a 4 AFC staircase procedure (see details in methods). The panel title shows the symmetry group's name. Horizontal yellow lines depict the chance level ( $25 \%$ correct discrimination).

\subsection{Experiment 2}

In Experiment 2, images from six symmetry groups were used: Group P1, having no internal structure, and groups P3, P3M1, P31M, P6, and P6M, which are all of a hexagonal lattice type. In this experiment the images of different sizes were presented to observers at different trials. These were complete images of the fully generated lattice and images cropped to sizes of $1 / 2 \times 1 / 2,1 \times 1,2 \times 2$, and $3 \times 3$ cells. Additionally, images of size $1 / 2 \times 1 / 2,1 \times 1$, and $2 \times 2$ cells were presented in one of two scales, the same scale as the full images or magnified by factor of 2 , such that one cell in a scaled image occupied the space on the computer screen equal to $2 \times 2$ of the original cells. The experiment was 
performed using a fixed stimulus design to improve the precision of performance estimates. The sets of thermodynamic temperatures were selected from Experiment 1, spanning approximately the active part of the psychometric function.

The results from symmetry group P1 are shown in Figure 2. Note that the performance for $3 \times 3$ cell textures (a solid orange curve) is practically the same as the performance for $8 \times 8$ cell textures (black solid curves) for three observers. Observer O4 performed many fewer trials ( $\mathrm{N}=23$ per condition); thus, their estimates are noisy. In contrast, all other conditions led to a chance level performance for all thermodynamic temperatures. These results imply that $2 \times 2$ repetitions are not sufficient to perceive a structure in a brief presentation, whereas $3 \times 3$ repetitions are as good as more repetitions for most observers. Note that the scaled textures with $2 \times 2$ cells (dashed red curves) occupy larger visual angles than the $3 \times 3$ cell textures do (orange solid curve). This result implies that the number of repetitions (content) is the critical parameter governing discrimination, not the physical size of the stimulus. Note the "all or none" behavior with respect to the number of repetitions, with the $2 \times 2$ psychometric curves being flat at a chance level, and the $3 \times 3$ curves practically overlapping with those of the full $(8 \times 8)$ stimulus. Thus, the transition seems to take place at a repetition number that is independent of temperature (texture noise).
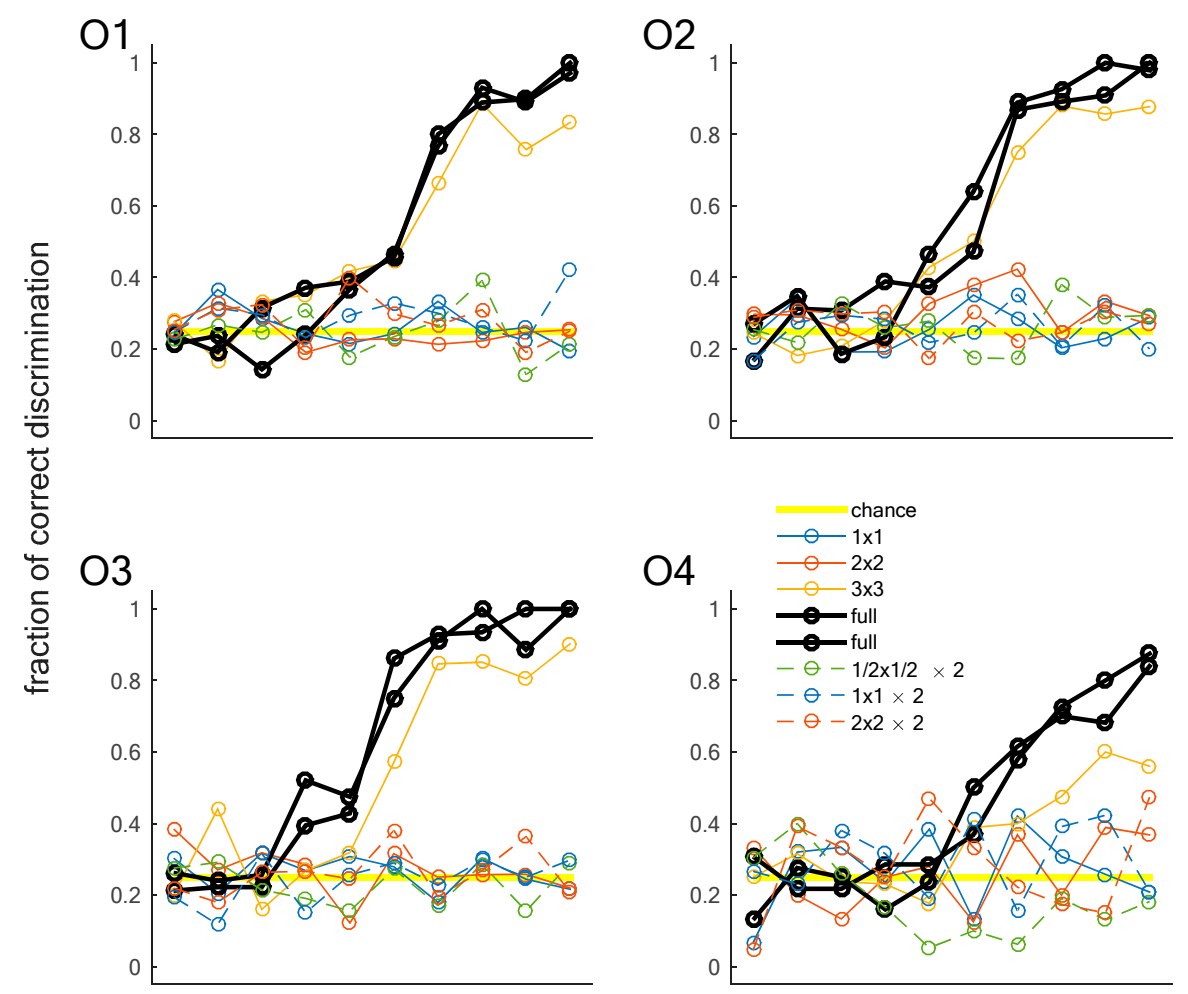

$\beta$

Figure 2. Human performance on the $4 \mathrm{AFC}$ discrimination task with textures from symmetry group P1 (only repetition is present), as a function of inverse thermodynamic temperature $\beta$. Different numbers of cells were presented to 4 human observers (O1-O4), as depicted in the legend. Dashed curves represent performance with stimuli spatially scaled up by a factor of 2 . The legend shows the number of repetitions of the basic motif presented in the texture for perfectly symmetric images (low thermodynamic temperature), denoted as $\mathrm{N} \times \mathrm{N}$, with "full" corresponding to $8 \times 8$ repetitions in group P1. This latter condition (P1) was presented twice and can be used as andication of the estimation precision. One can observe that the performance for $3 \times 3$ cell textures is similar to the performance for $8 \mathrm{X} 8$ cell textures, whereas all other conditions lead to chance level performance.

The results from all six groups for one observer are presented in Figure 3, whereas the results for the other three observers are shown in Appendix A. Psychometric curves for the same symmetry 
group but different image manipulations (such as cropping and scaling) are presented in a single panel. The results obtained from images drawn from group P1 (having no internal symmetry) are described above. The other five symmetry groups used in Experiment 2 were generated with cells having an internal structure. One can see that images from these groups cut to a $2 \times 2$ cell size are discriminated better than chance at low temperatures (red curves), implying that the internal structure of the cell provides additional information for the discrimination. Moreover, the original images (solid lines) and their physically scaled (x2) versions (dashed lines) yield practically identical psychometric curves, indicating that the content rather than the physical size determines performance in this task.

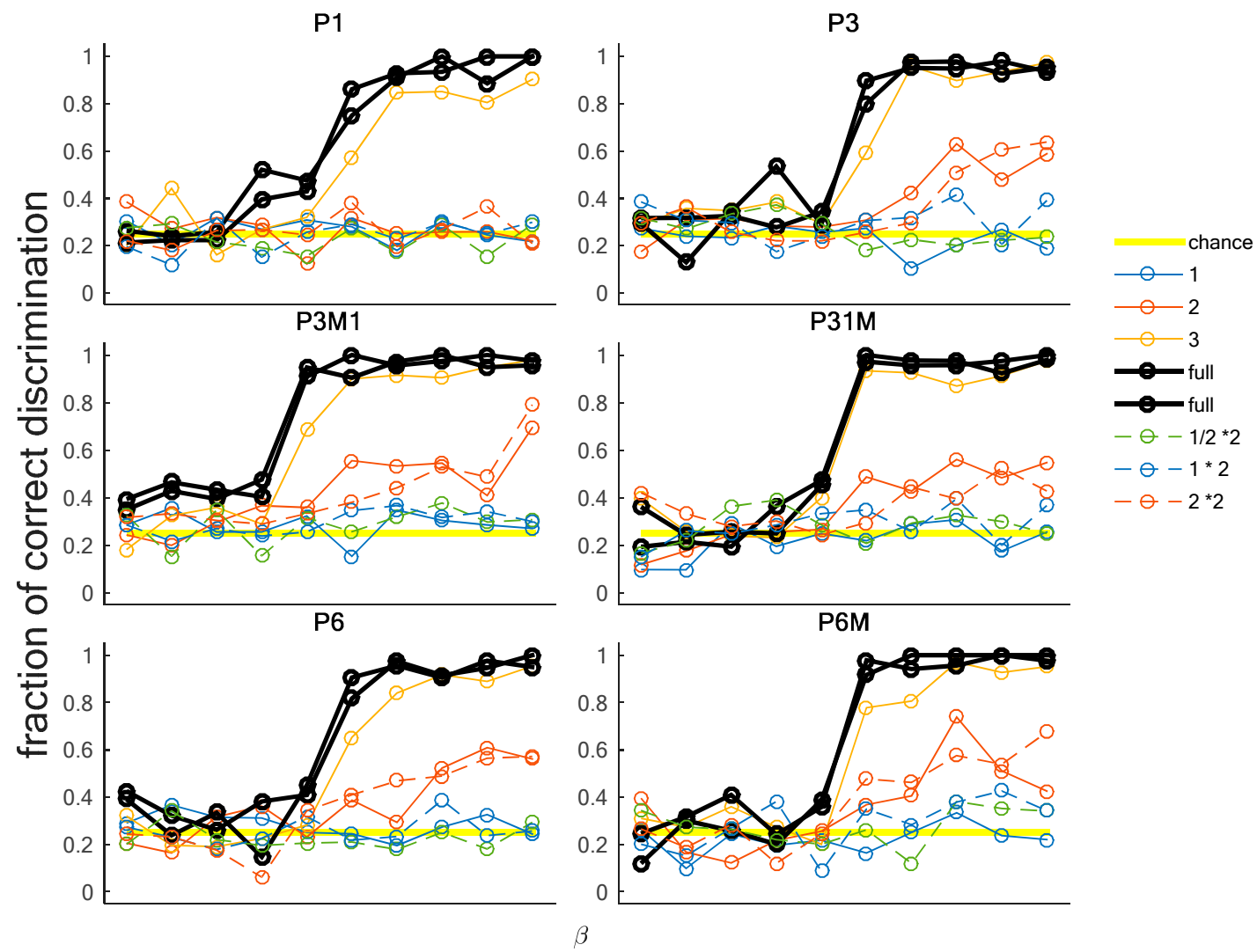

Figure 3. Results of Experiment 2 for Observer O1. Images from six groups (the group name is in the panel title) were presented in this experiment. Psychometric curves for a single group are shown in each panel. Solid lines represent psychometric curves with their original image size and dashed curves with the same color represent conditions with images physically magnified by a factor of 2 (see Methods). The following conditions were presented: A fully generated lattice, containing $8 \times 8$ cells (black lines), images cropped to a size of $3 \times 3$ cells (orange lines), $2 \times 2$ cells (red lines), one cell (blue line), and a $1 / 2 \times 1 / 2$ cell (green line).

Note that the performance could not be compared across conditions, since potentials defined for different groups include different numbers of symmetries, leading to a different number of terms in Equation (3) and the dependency on these terms is highly nonlinear. However, the performance can be compared across conditions for the same symmetry group. For example, unlike the "all or none" behavior observed with $\mathrm{P} 1$, one can observe a gradual increase in performance with an increasing number of repetitions in groups having internal symmetry. For the latter, the onset of deviation from a chance level of the red psychometric curves starts at a lower temperature than that of the black and orange curves (curves shifted right). This may indicate different and possibly independent mechanisms underlying discrimination under those conditions that involve different kinds of symmetries. One possibility is that for a large enough number of repetitions the "repetition detector" effectively dominates, whereas for $2 \times 2$ cells the mechanisms responsible for some of the 
internal symmetries play a major role in the discrimination performance, since in the absence of internal symmetry performance (group P1), for all temperatures, they are at chance level.

\section{Discussion}

Here we studied the perceptual integration of visual textures having varying degrees of order. To this end, we have designed stimuli ranging from symmetrical repetitive images to images having a completely random structure. More specifically, by varying the thermodynamic temperature in the Boltzmann distribution for artificial physical systems and by simulating these systems, we obtained images corresponding to thermal equilibrium. The resulting images were repetitive at lower temperatures and random for higher temperatures. Moreover, the textural elements were of the same quality for all images compared to classical studies where images were degraded by adding white noise. By choosing appropriate potentials in the Boltzmann distribution, we obtained ordered images corresponding to all 17 wallpaper groups of symmetry [18]. Psychometric curves measuring discrimination performance as a function of inverse thermodynamic temperature were consistent across observers. This result suggests that the human visual system has mechanisms sensitive to the degree of order in images. By cropping images from group P1 (ordered images having only repetition symmetry) to a different number of repetitions, we found that discrimination performance became saturated at $3 \times 3$ repetitions. For instance, a larger number of repetitions led to the same discrimination performance, whereas a smaller number of repetitions $(2 \times 2)$ led to a chance performance. This result suggests that the order is effectively computed using information from a limited spatial extent. However, when images containing more symmetries were presented, the discrimination performance for $2 \times 2$ repetitions were above chance level for low temperatures. This indicates that other symmetry detection mechanisms, probably local, are also involved in order perception, possibly competing with repetition detection mechanisms. Furthermore, physical scaling of the images does not alter discrimination performance, indicating that the number of repetitions and not the physical size plays an important role in order perception.

The stimuli we used are images from artificial 2D physical systems in thermal equilibrium at different temperatures. It is known from statistical physics that similar systems undergo continuous phase transition from a disordered state to an ordered one when the temperature changes. It is important that there is a specific temperature (a critical temperature) where the symmetry of the system changes. More specifically, above a critical temperature, the physical system becomes disordered (random) and symmetry gradually builds below a critical temperature. This feature of the stimuli enables a fine-grained study of the sensitivity to a specific symmetry or to symmetry combinations. Moreover, the degree of symmetry can be independently quantified using statistical physics tools based on the images only. What is more important is that the textural elements are the same for all textures; this allows one to select textural elements with well-characterized response properties in the early visual cortex. This would enable electrophysiological studies of textural integration in order to measure psychometric functions, or to compare the threshold against temperature curves to characterize order (symmetry or a combination of symmetries) detection or discrimination.

The relatively small integration size is consistent with recent findings that the processing of symmetrical patterns [11,23] and visual textures [24] lead to a larger blood-oxygen-level dependent response in area V2/V3 and later areas. The absence of response in area V1 in those studies may indicate that order perception mechanisms operate based on the output of localized bandlimited mechanisms, which are well characterized in area V1. This would suggest that adding noise to every pixel would degrade the signal-to-noise ratio at an early stage, and consequently, degrade symmetry detection, as was observed by Reference [13]. Here we used a different kind of noise-thermal noise, which permuted the textural elements and left the signal-to-noise ratio fixed at an early perceptual stage where visual processing is local.

It is interesting that in the recognition of visual objects, human observers exhibited a sharp drop in performance when the degree of information is decreased, whereas the size of the image 
is preserved [25], suggesting a phase transition. If symmetry detectors are essential elements of object recognition, the sharp drop in object categorization performance with a decreased amount of information suggests that with a small amount of information the symmetry information would be lost and patches would be perceived either as random or part of textures; in other words, there would be too many interpretations of an image as an object.

\section{Conclusions}

We showed that: (1) $3 \times 3$ repetitions of the motif are as effective as a larger number of repetitions for repetitive order detection, (2) the internal structure within the repetition cell provides additional information for discrimination, and (3) the number of repetitions is important for the discrimination, and not the physical size of the stimulus. These findings impose constraints on possible models for symmetry perception. More specifically, the integration is most probably local and scale invariant. The method we used allows detailed psychophysical and electrophysiological studies of integration mechanisms, leaving the response of the early visual cortex unchanged unless it is involved in symmetry processing.

Author Contributions: M.K. and D.S. design of study; M.K. presentation and analysis software, H.H. data collection. All authors wrote paper.

Funding: This work was supported by the Basic Research Foundation administered by the Israel Academy of Sciences and Humanities (grant \#1056/15) and by The Weizmann Braginsky Center for the Interface between the Sciences and the Humanities.

Acknowledgments: We would like to thank Anthony Norcia for suggesting to consider the wallpaper symmetry groups.

Conflicts of Interest: The authors declare no conflicts of interest.

\section{Appendix A}

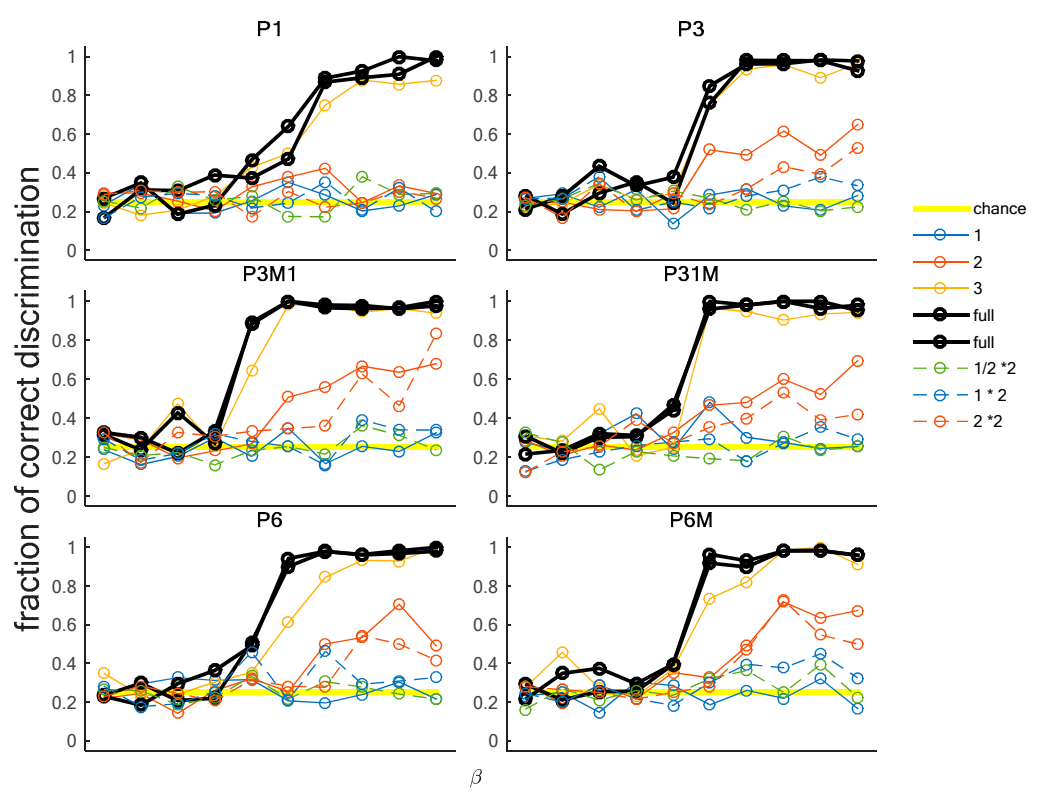

Figure A1. Results of Experiment 2 for Observer O2. 


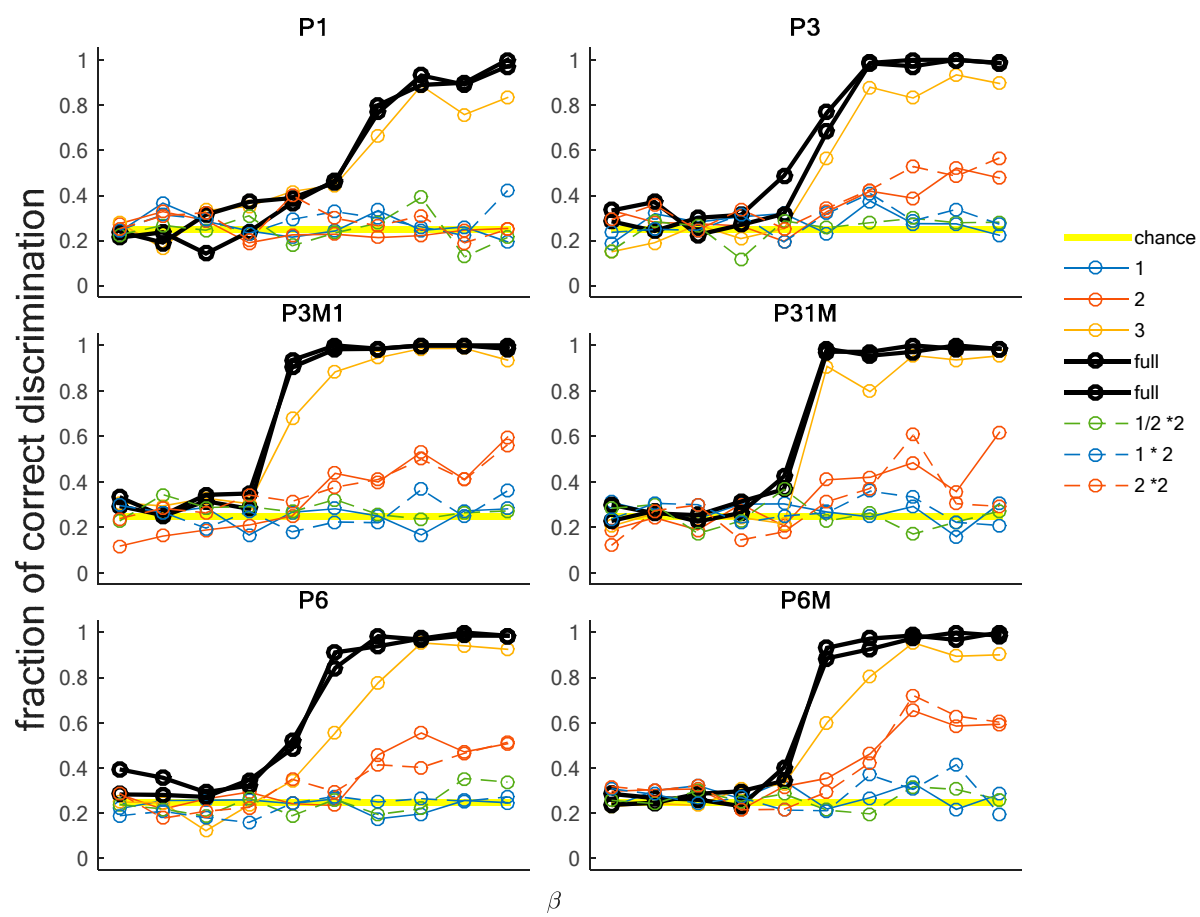

Figure A2. Results of Experiment 2 for Observer O3.
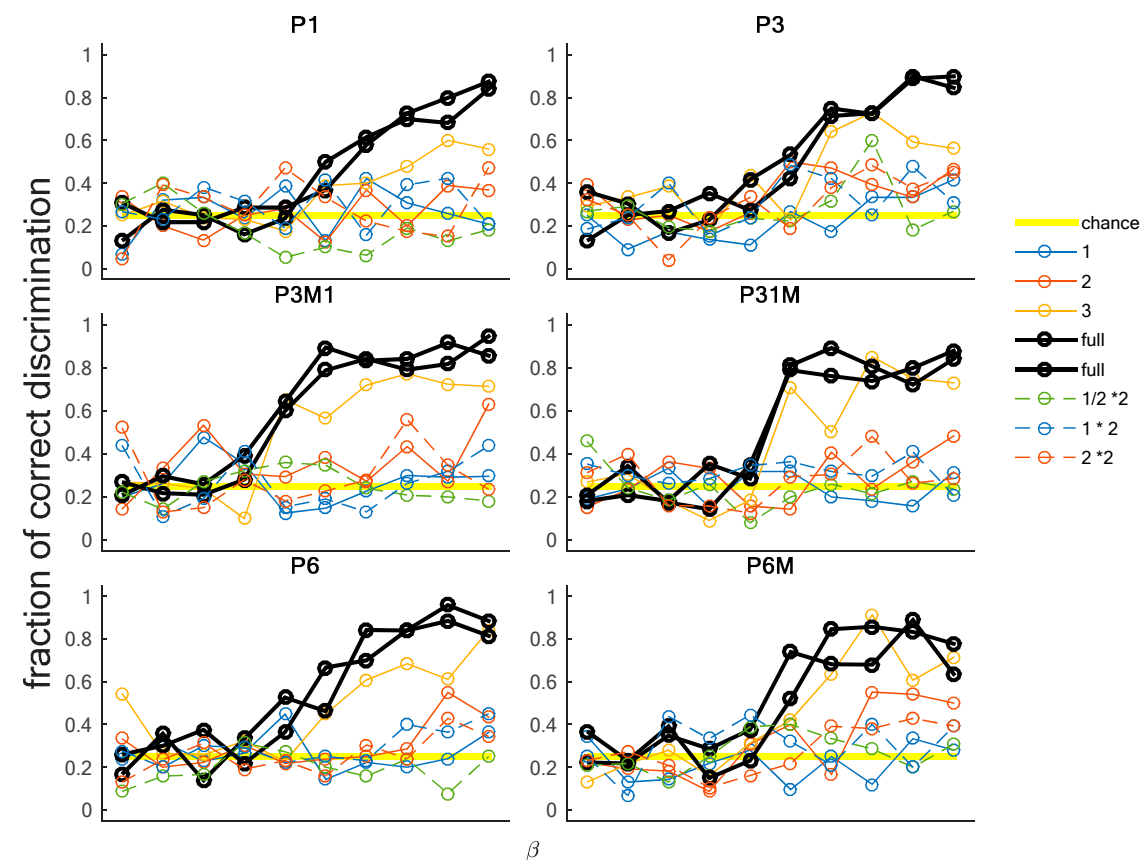

Figure A3. Results of Experiment 2 for Observer O4.

\section{References}

1. Hubel, D.H.; Wiesel, T.N. Receptive fields, binocular interaction and functional architecture in the cat's visual cortex. J. Physiol. 1962, 160, 106-154. [CrossRef] [PubMed]

2. Hubel, D.H.; Wiesel, T.N. Receptive fields of optic nerve fibres in the spider monkey. J. Physiol. 1960, 154, 572-580. [CrossRef] [PubMed]

3. Julesz, B. Visual Pattern Discrimination. IRE Trans. Info. Theory 1962, 8, 84-92. [CrossRef]

4. Julesz, B. Texture and visual perception. Sci. Am. 1965, 212, 38-48. [CrossRef] [PubMed] 
5. Caelli, T.; Julesz, B.; Gilbert, E. On perceptual analyzers underlying visual texture discrimination: Part II. Biol. Cybern. 1978, 29, 201-214. [CrossRef] [PubMed]

6. Portilla, J.; Simoncelli, E.P. A parametric texture model based on joint statistics of complex wavelet coefficients. Intl. J. Comp. Vis. 2000, 40, 49-71. [CrossRef]

7. Klein, S.A.; Tyler, C.W. Phase discrimination of compound gratings: generalized autocorrelation analysis. J. Opt. Soc. Am. A 1986, 3, 868. [CrossRef] [PubMed]

8. Julesz, B.; Gilbert, E.N.; Shepp, L.A.; Frisch, H.L. Inability of humans to discriminate between visual textures that agree in second-order statistics-revisited. Perception 1973, 2, 391-405. [CrossRef] [PubMed]

9. Kubovy, M. The perceptual organization of dot lattices. Psychon Bull. Rev. 1994, 1, 182-190. [CrossRef] [PubMed]

10. Wagemans, J.; Elder, J.H.; Kubovy, M.; Palmer, S.E.; Peterson, M.A.; Singh, M.; von der Heydt, R. A century of Gestalt psychology in visual perception: I. Perceptual grouping and figure-ground organization. Psychol. Bull. 2012, 138, 1172-1217. [CrossRef] [PubMed]

11. Kohler, P.J.; Clarke, A.; Yakovleva, A.; Liu, Y.; Norcia, A.M. Representation of maximally regular textures in human visual cortex. J. Neuros. 2016, 36, 714-729. [CrossRef] [PubMed]

12. Schattschneider, D. The plane symmetry groups: their recognition and notation. Am. Math. Mon. 1978, 85, 439. [CrossRef]

13. Tyler, C.W.; Chang, J.J. Visual echoes: The perception of repetition in quasi-random patterns. Vision Res. 1977, 17, 109-116. [CrossRef]

14. Katkov, M.; Harris, H.; Sagi, D. Visual perception of order-disorder transition. Front. Psychol. 2015, 6, 734. [CrossRef] [PubMed]

15. Landau, L.D. On the theory of phase transitions. Zh. Eks. Teor. Fiz. 1937, 11, 19-32. [CrossRef]

16. Sethna, J.P. Statistical Mechanics: Entropy, Order Parameters, and Complexity; Oxford University Press: New York, NY, USA, 2006; ISBN 978-0198566779.

17. Brush, S. History of the lenz-ising model. Rev. Mod. Phys. 1967, 39, 883-893. [CrossRef]

18. Liu, Y.; Hel-Or, H.; Kaplan, C.S.; Gool, L. Van computational symmetry in computer vision and computer graphics. Found. Trends®Comput. Graph. Vis. 2009, 5, 1-195. [CrossRef]

19. Bravais, A. Mémoire sur les sytèmes formés par des points distribués régulièrement sur un plan ou dans l'espace (in France). J. l'École Polytech. Paris 1850, 19, 1-128.

20. Katkov, M. Wallpaper Group Colored Gibbs Sampler 2018. Available online: https://github.com/mkatkov / WallpaperColoredGibbsSampler (accessed on 17 October 2018).

21. Casella, G.; George, E.I. Explaining the Gibbs sampler. Am. Stat. 1992, 46, 167. [CrossRef]

22. Gonzalez, J.; Low, Y.; Gretton, A.; Guestrin, C. Parallel GIBBS SAMPLING: From Colored Fields to Thin Junction Trees. Proc. Mach. Learn. Res. 2011, 15, 324-332.

23. Kohler, P.J.; Cottereau, B.R.; Norcia, A.M. Dynamics of perceptual decisions about symmetry in visual cortex. Neuroimage 2018, 167, 316-330. [CrossRef] [PubMed]

24. Freeman, J.; Ziemba, C.M.; Heeger, D.J.; Simoncelli, E.P.; Movshon, J.A. A functional and perceptual signature of the second visual area in primates. Nat. Neurosci. 2013, 16, 974-981. [CrossRef] [PubMed]

25. Ullman, S.; Assif, L.; Fetaya, E.; Harari, D. Atoms of recognition in human and computer vision. Proc. Natl. Acad. Sci. USA 2016, 113, 2744-2749. [CrossRef] [PubMed]

(c) 2018 by the authors. Licensee MDPI, Basel, Switzerland. This article is an open access article distributed under the terms and conditions of the Creative Commons Attribution (CC BY) license (http://creativecommons.org/licenses/by/4.0/). 